\title{
THE ASYMPTOTIC \\ BROWDER HARTMAN STAMPACCHIA CONDITION AND INTERIOR BANDS OF $\varepsilon$-SOLUTIONS FOR NONLINEAR COMPLEMENTARITY PROBLEMS
}

\author{
G. ISAC AND S.Z. NÉMETH
}

\begin{abstract}
In this paper we study two remarkable properties of the interior band of $\varepsilon$-solutions of a general nonlinear complementarity problem in the $n$-dimensional Euclidean space. The study is based on the notion of scalar derivative, on the notion of infinitesimal interior point $\varepsilon$-exceptional family of elements for a function and on the asymptotic Browder Hartman Stampacchia condition.
\end{abstract}

1. Introduction. Let $\left(\mathbf{R}^{n},\langle\cdot, \cdot\rangle\right)$ be the $n$-dimensional Euclidean space ordered by the closed pointed convex cone $\mathbf{R}_{+}^{n}$ and $f: \mathbf{R}^{n} \rightarrow \mathbf{R}^{n}$ a continuous function. We consider the following nonlinear complementarity problem:

$$
N C P\left(f, \mathbf{R}_{+}^{n}\right):\left\{\begin{array}{l}
\text { find } x^{0} \in \mathbf{R}_{+}^{n} \text { such that } \\
f\left(x^{0}\right) \in \mathbf{R}_{+}^{n} \text { and }\left\langle x^{0}, f\left(x^{0}\right)\right\rangle=0 .
\end{array}\right.
$$

It is well known that the $N C P\left(f, \mathbf{R}_{+}^{n}\right)$ has many applications in optimization, economics, engineering, game theory and mechanics $[\mathbf{6}$, $10,14,16,17]$.

We note that there exist several equivalent formulations of the $N C P\left(f, \mathbf{R}_{+}^{n}\right)$. In particular, several formulations are in the form of a nonlinear equation of the form $F(x)=0$, where $F: \mathbf{R}^{n} \rightarrow \mathbf{R}^{n}$ is a continuous function. By using such formulations, several techniques proposed by some authors are based on the idea to perturb $F$ to a certain $F(x, \varepsilon)$, where $\varepsilon$ is a positive parameter and to consider the equation $F(x, \varepsilon)=0$. If $F(x, \varepsilon)=0$ has a unique solution,

AMS Mathematics subject classification. Primary 54B20, 54F15.

Keywords and phrases. Scalar derivative, interior band of $\varepsilon$-solutions, asymptotic Browder Hartman Stampacchia condition, complementarity problems.

The second author was supported by Hungarian Research Grant OTKA T043276 and T043241.

Received by the editors on June 13, 2005.

Copyright (C)2007 Rocky Mountain Mathematics Consortium 
denoted by $x(\varepsilon)$ and $x(\varepsilon)$ is continuous in $\varepsilon$, then the solutions describe (depending on the properties of $F$ ) a short path denoted by $\left.\left.\{x(\varepsilon): \varepsilon \in] 0, \varepsilon_{0}\right]\right\}$ or a long path $\{x(\varepsilon): \varepsilon \in] 0, \infty[\}$. We note that, if a short path $\left.\left.\{x(\varepsilon): \varepsilon \in] 0, \varepsilon_{0}\right]\right\}$ is bounded, then for any sequence $\left\{\varepsilon_{k}\right\}$ with $\left\{\varepsilon_{k}\right\} \rightarrow 0$, the sequence $\left\{x\left(\varepsilon_{k}\right)\right\}$ has at least one accumulation point, which by continuity is a solution to the $N C P\left(f, \mathbf{R}_{+}^{n}\right)$. Based on this fact, several numerical methods for solving the $N C P\left(f, \mathbf{R}_{+}^{n}\right)$ have been developed, as for example the interior point path-following methods, regularization methods and noninterior path-following methods among others. About such methods the reader can see the papers $[\mathbf{1}, \mathbf{2}, \mathbf{3}-\mathbf{5}, \mathbf{8}-\mathbf{1 5}, \mathbf{2 1}-\mathbf{2 5}, \mathbf{2 8}]$. The most common interior point pathfollowing method is based on the notion of central path. We recall [29] that the curve $\{x(\varepsilon): \varepsilon \in] 0, \infty[\}$ is said to be the central path if for each $\varepsilon>0$ the vector $x(\varepsilon)$ is the unique solution to the system

$$
\left\{\begin{array}{l}
x(\varepsilon)>0, f(x(\varepsilon))>0 \\
\text { and } X(\varepsilon) f(x(\varepsilon))=\varepsilon e,
\end{array}\right.
$$

where the inequality " $>$ " means that the components of the vector are strictly positive, $e=(1, \ldots, 1)^{T}, X(\varepsilon)=$ the matrix $\operatorname{diag}(x(\varepsilon))$ and $x(\varepsilon)$ is continuous on $] 0, \infty[$. It is well known that, for a general $N C P\left(f, \mathbf{R}_{+}^{n}\right)$, the system (1) may have multiple solutions for a given $\varepsilon>0$, and even if the solution is unique it is not necessarily continuous in $\varepsilon$. Therefore, the existence of the central path is not always guaranteed.

We consider in this paper the multi-valued mapping $\mathcal{U}:] 0, \infty[\rightarrow$ $\mathcal{S}\left(\mathbf{R}_{++}^{n}\right)$ defined by

$$
\mathcal{U}(\varepsilon)=\left\{x \in \mathbf{R}_{++}^{n}: f(x)>0, X f(x)=\varepsilon e\right\},
$$

where $X=$ the matrix $\operatorname{diag}(x), \mathcal{S}\left(\mathbf{R}_{++}^{n}\right)$ is the collection of all subsets of $\mathbf{R}_{++}^{n}$ and $\mathbf{R}_{++}^{n}=\left\{x=\left(x_{1}, \ldots, x_{n}\right): x_{1}>0, \ldots, x_{n}>0\right\}$. We say that $\mathcal{U}$ is the interior band mapping. The multi-valued mapping $\mathcal{U}$ was studied from several points of view in [29].

Now, the main goal of our paper is to study under what conditions the multi-valued mapping $\mathcal{U}$ has the following properties:

(a) $\mathcal{U}(\varepsilon) \neq \varnothing$ for each $\varepsilon \in] 0, \infty[$.

(b) For any fixed $\varepsilon_{0}>0$, the set $\cup_{\varepsilon \in] 0, \varepsilon_{0}} \mathcal{U}(\varepsilon)$ is bounded. 
Conditions (a) and (b) were defined in [29].

Our study is based on the asymptotic Browder Hartman Stampacchia condition, on the notion of scalar derivative and the notion of infinitesimal interior point $\varepsilon$-exceptional family of elements for the inversion of $f$. By the results presented in this paper we show another utility of the Browder Hartman Stampacchia condition, never put in evidence by other authors.

\section{Preliminaries}

For more details about the notions and the results presented in this section, the reader is referred to $[\mathbf{2 9}]$.

Definition 2.1. Let $f: \mathbf{R}^{n} \rightarrow \mathbf{R}^{n}$ be a continuous function. Given a scalar $\varepsilon>0$ we say that a family $\left\{x^{r}\right\}_{r>0} \subset \mathbf{R}_{++}^{n}$ is an interior point $\varepsilon$-exceptional family for $f$ if $\left\|x^{r}\right\| \rightarrow \infty$ as $r \rightarrow \infty$ and for each $x^{r}$ there exists a positive number $0<\mu_{r}<1$ such that

$$
f_{i}\left(x^{r}\right)=\frac{1}{2}\left(\mu_{r}-\frac{1}{\mu_{r}}\right) x_{i}^{r}+\frac{\varepsilon \mu_{r}}{x_{i}^{r}} \quad \text { for all } \quad i=1,2, \ldots, n .
$$

Theorem 2.1. Let $f: \mathbf{R}^{n} \rightarrow \mathbf{R}^{n}$ be a continuous function. Then for each $\varepsilon>0$ there exists either a point $x(\varepsilon)$ such that

$$
x(\varepsilon)>0, \quad f(x(\varepsilon))>0, \quad x_{i}(\varepsilon) f_{i}(x(\varepsilon))=\varepsilon, \quad i=1,2, \ldots, n .
$$

or an interior point $\varepsilon$-exceptional family for $f$.

Proof. This result is proved in $[\mathbf{2 9}]$.

Let $K \subset \mathbf{R}^{n}$ be a closed pointed convex cone and $f: \mathbf{R}^{n} \rightarrow \mathbf{R}^{n}$ a continuous function. The following notion was used in $[\mathbf{1 7}, \mathbf{1 8}]$.

Definition 2.2. We say that a family of elements $\left\{x^{r}\right\}_{r>0} \subset K$ is an exceptional family of elements, denoted by EFE, for $f$ with respect to $K$ if $\left\|x^{r}\right\| \rightarrow \infty$ as $r \rightarrow \infty$ and for every real number $r>0$, there 
exists a real number $\mu_{r}>0$ such that the vector $u^{r}=\mu_{r} x^{r}+f\left(x^{r}\right)$ satisfies the following conditions:

(1) $u^{r} \in K^{*}$ (the dual of $\left.K\right)$,

(2) $\left\langle u^{r}, x^{r}\right\rangle=0$.

If in the definition of the problem $N C P\left(f, \mathbf{R}_{+}^{n}\right)$ we replace the cone $\mathbf{R}_{+}^{n}$ by the cone $K$, we obtain the problem $N C P(f, K)$, i.e.,

$$
N C P(f, K):\left\{\begin{array}{l}
\text { find } x^{0} \in K \text { such that } \\
f\left(x^{0}\right) \in K^{*} \text { and }\left\langle x^{0}, f\left(x^{0}\right)\right\rangle=0 .
\end{array}\right.
$$

Theorem 2.2. If $f: \mathbf{R}^{n} \rightarrow \mathbf{R}^{n}$ is a continuous function and $K \subset \mathbf{R}^{n}$ is a closed pointed convex cone, then there exists either a solution to the $N C P(f, K)$ or an exceptional family of elements for $f$ with respect to $K$.

Proof. A proof of this result is given in [17].

The following definition and theorem can be found in [17].

Definition 2.3. Let $(H,\langle\cdot, \cdot\rangle)$ be a Hilbert space, $K \subset H$ a closed convex cone and $f: H \rightarrow H$ a mapping. We say that the mapping $f$ satisfies condition $\Theta$ with respect to $K$ if

$$
\left\{\begin{array}{l}
\text { there exists } \rho>0 \text { such that for each } x \in K \text { with }\|x\|>\rho \\
\text { there exists } p \in K \text { with }\|p\|<\|x\| \text { such that } \\
\langle x-p, f(x)\rangle \geq 0
\end{array}\right.
$$

Theorem 2.3. Let $H$ be a Hilbert space, $K \subset H$ a closed convex cone and $f: H \rightarrow H$ a mapping. If $f$ satisfies condition $\Theta$ with respect to $K$, then it is without exceptional family of elements with respect to $K$.

3. The Browder Hartman Stampacchia condition. First we recall a general classical result. Let $(E,\|\cdot\|)$ be a reflexive Banach space 
and $f: E \rightarrow E^{*}$. We say that $f$ is hemi-continuous if it is continuous from the line segment to the weak topology of $E^{*}$.

We say that $f$ is monotone if for any $x, y \in E$ we have that

$$
\langle x-y, f(x)-f(y)\rangle \geq 0,
$$

and we say that $f$ is strongly monotone if there exists a constant $\alpha>0$ such that for any $x, y \in E$ we have

$$
\langle x-y, f(x)-f(y)\rangle \geq \alpha\|x-y\|^{2} .
$$

Definition 3.1. We say that $f$ satisfies the Browder Hartman Stampacchia condition, denoted by BHS, on a closed convex cone $K \subset E$ if there exists $\rho>0$ such that $\langle x, f(x)\rangle>0$, for any $x \in K$ with $\|x\|=\rho$.

The following result is to support the importance of condition (BHS).

Theorem 3.1 (Browder Hartman Stampacchia). Let $(E,\|\cdot\|)$ be a reflexive Banach space, $f: E \rightarrow E^{*}$ a monotone hemi-continuous mapping and $K \subset E$ a closed convex cone. If $f$ satisfies condition BHS on $K$, then the problem $N C P(f, K)$ has a solution.

Proof. A proof of this result is given in [17, Theorem 4.6].

Proposition 3.1. If $f: E \rightarrow E^{*}$ is strongly monotone on $K$, then $f$ satisfies condition BHS on $K$.

Proof. Indeed, if we take $y=0$, we have

$$
\langle x, f(x)\rangle \geq\langle x, f(0)\rangle+\alpha\|x\|^{2} .
$$

If $f(0)=0$ we deduce that $\langle x, f(x)\rangle \geq \alpha\|x\|^{2}$, for any $x \in K$. If we take an arbitrary $\rho>0$, we have that $\langle x, f(x)\rangle>0$, for any $x \in K$ with $\|x\|=\rho$. 
If $f(0) \neq 0$, then in this case we consider the set

$$
D=\left\{x \in K:\|x\| \leq \frac{\|f(0)\|}{\alpha}\right\}
$$

which is nonempty and bounded. For any $x \in K \backslash D$ we have

$$
\alpha\|x\|^{2}>\|x\| \cdot\|f(0)\| \geq-\langle x, f(0)\rangle
$$

which implies $\langle x, f(x)\rangle>0$ for any $x \in K \backslash D$. Because $D$ is bounded, there exists $\rho>0$ such that $D \subset B(0, \rho)$ and for any $x \in K$ with $\|x\|=\rho$ we have $\langle x, f(x)\rangle>0$.

By the next result we can obtain many functions which satisfy condition (BHS). Let $K \subset \mathbf{R}^{n}$ be a closed convex cone. We say that a function $T: K \rightarrow \mathbf{R}^{n}$ satisfies condition $(\beta)$ if there exists a real number $\beta(T)>0$ such that for all $x \in K$ with $\|x\| \geq 1$, we have $\|T(x)\| \leq \beta(T)\|x\|$.

Examples. (1) Any linear continuous operator $T: \mathbf{R}^{n} \rightarrow \mathbf{R}$ satisfies condition $(\beta)$.

(2) If $T: K \rightarrow \mathbf{R}^{n}$ is a $k$-Lipschitz mapping, then $T$ satisfies condition $(\beta)$ with $\beta(T)=k+\beta_{0}$, where $\beta_{0}=k\left\|x^{0}\right\|+\left\|T\left(x^{0}\right)\right\|$ and $x^{0}$ is an arbitrary element in $K$.

Theorem 3.2. Let $f: K \rightarrow \mathbf{R}^{n}$ be a continuous function and $T: K \rightarrow \mathbf{R}^{n}$ a function satisfying condition $(\beta)$. If the following conditions are satisfied:

(1) $\liminf _{\|x\| \rightarrow \infty}\langle f(x)-T(x), x\rangle /\|x\|^{2} \geq k_{0}>0$,

(2) $\beta(T)<k_{0}$,

then there exists $\rho>0$ such that $\langle x, f(x)\rangle>0$, for all $x \in K$ with $\|x\|=\rho$.

Proof. Let $\varepsilon>0$ be such that $\beta(T)+\varepsilon<k_{0}$. From assumption (1) we have that there exists $\rho_{0}>0$ such that for all $x \in K$ with $\|x\|>\rho_{0}$ we have

$$
\frac{\langle f(x)-T(x), x\rangle}{\|x\|^{2}}>k_{0}-\varepsilon,
$$


which implies

$$
\langle f(x)-T(x), x\rangle>\left(k_{0}-\varepsilon\right)\|x\|^{2}
$$

and finally,

$$
\langle f(x), x\rangle>\langle T(x), x\rangle+\left(k_{0}-\varepsilon\right)\|x\|^{2} .
$$

From the last inequality we obtain

$$
\langle f(x), x\rangle \geq-\beta(T)\|x\|^{2}+\left(k_{0}-\varepsilon\right)\|x\|^{2}=\|x\|^{2}\left(-\beta(T)-\varepsilon+k_{0}\right)>0,
$$

for all $x \in K$ with $\|x\|>\rho_{0}$. If we take $\rho>\rho_{0}$, the proof is complete.

Remark 3.1. Theorem 3.2 is applicable in the following cases

(i) $f(x)=T(x)+a x+b$, where $a>0, b \in \mathbf{R}^{n}$ is an arbitrary vector and $T$ satisfies condition $(\beta)$ with $\beta(T)<a$.

(ii) $f(x)=T(x)+L(x)+b$, where $b \in \mathbf{R}^{n}$ is an arbitrary vector, $L$ is a linear operator from $\mathbf{R}^{n}$ into $\mathbf{R}^{n}$ such that $\langle L(x), x\rangle \geq k_{0}\|x\|^{2}$, for any $x \in K$ and $T$ satisfies condition $\beta$ with $\beta(T)<k_{0}$.

In the $n$-dimensional Euclidean space Theorem 3.1 has the following form:

Theorem 3.3. Let $K \subset \mathbf{R}^{n}$ be a closed convex cone and $f: \mathbf{R}^{n} \rightarrow$ $\mathbf{R}^{n}$ a continuous function. If there exists $\rho>0$ such that $\langle x, f(x)\rangle \geq 0$ for any $x \in K$ with $\|x\|=\rho$, then the problem $N C P(f, K)$ has a solution $x^{*}$ such that $\left\|x^{*}\right\| \leq \rho$.

Proof. Let $T_{\rho}$ be the radial retraction onto the ball $B(0, \rho)=\{x \in$ $\left.\mathbf{R}^{n}:\|x\| \leq \rho\right\}$, i.e.,

$$
T_{\rho}(x)= \begin{cases}x & \text { if }\|x\| \leq \rho \\ \rho x /\|x\| & \text { if }\|x\|>\rho .\end{cases}
$$

It is known that $T_{\rho}$ is continuous. If we denote by $K_{\rho}=B(0, \rho) \cap K$, we have that $T_{\rho}$ is also a continuous retraction of the cone $K$ onto $K_{\rho}$.

We denote by $F: \mathbf{R}^{n} \rightarrow \mathbf{R}^{n}$ the continuous mapping defined by

$$
F(x)=f\left(T_{\rho}(x)\right)+\left\|x-T_{\rho}(x)\right\| x .
$$


For any $x \in K$ with $\|x\|>\rho$ we have $\langle x, F(x)\rangle>0$. Indeed, we have

$$
\begin{aligned}
\langle x, F(x)\rangle & =\left\langle x, f\left(T_{\rho}(x)\right)\right\rangle+\left\|x-T_{\rho}(x)\right\| \cdot\|x\|^{2} \\
& =\left\langle\frac{\|x\|}{\rho} T_{\rho}(x), f\left(T_{\rho}(x)\right)\right\rangle+\left\|x-T_{\rho}(x)\right\| \cdot\|x\|^{2}>0 .
\end{aligned}
$$

Because of the fact that $\langle x, F(x)\rangle>0$ for any $x \in K$ with $\|x\|>\rho$, it is easy to show that $F$ satisfies condition $\Theta$ with respect to $K$. Hence, by Theorem 2.3, $F$ is without EFE with respect to $K$. Applying Theorem 2.2, we have that $N C P(F, K)$ has a solution $x^{*}$ which must satisfy the inequality $\left\|x^{*}\right\| \leq \rho$. Therefore, $F\left(x^{*}\right)=f\left(x^{*}\right)$ and $x^{*}$ is a solution to $N C P(f, K)$.

Remark 3.2. Theorem 3.3 is known in complementarity theory, but our proof presented here is different than other proofs.

Remark 3.3. If $f: \mathbf{R}^{n} \rightarrow \mathbf{R}^{n}$ is a continuous function such that there exists $\rho>0$ with the property that $\langle x, f(x)\rangle \geq 0$ for any $x \in K$ with $\|x\|=\rho$, then the function $F(x)=f\left(T_{\rho}(x)\right)+\left\|x-T_{\rho}(x)\right\| x$ is such that

$$
\liminf _{\substack{\|x\| \rightarrow+\infty \\ x \in K}}\langle x, F(x)\rangle=+\infty .
$$

Indeed, we have, for any $x \in K$ with $\|x\|>\rho$,

$$
\begin{aligned}
\langle x, F(x)\rangle & =\left\langle x, f\left(T_{\rho}(x)\right)+\left\|x-T_{\rho}(x)\right\| \cdot\|x\|^{2}\right. \\
& =\frac{\|x\|}{\rho}\left\langle T_{\rho}(x), f\left(T_{\rho}(x)\right)\right\rangle+\left\|x-T_{\rho}(x)\right\| \cdot\|x\|^{2} \\
& \geq\left\|x-T_{\rho}(x)\right\| \cdot\|x\|^{2} \geq \rho^{2}\left\|x-T_{\rho}(x)\right\| \geq \rho^{2}[\|x\|-\rho] .
\end{aligned}
$$

Computing liminf $\operatorname{in}_{\substack{\| x \in+\infty \\ x \in K}}$, we obtain that

$$
\liminf _{\substack{\|x\| \rightarrow+\infty \\ x \in K}}\langle x, F(x)\rangle=+\infty
$$


4. The asymptotic Browder Hartman Stampacchia condition. Let $\left(\mathbf{R}^{n},\langle\cdot, \cdot\rangle\right)$ be the $n$-dimensional Euclidean space, $K \subset \mathbf{R}^{n}$ a closed pointed convex cone and $f: \mathbf{R}^{n} \rightarrow \mathbf{R}^{n}$ a continuous function. We introduce the following condition.

Definition 4.1. We say that $f$ satisfies the asymptotic Browder Hartman Stampacchia condition, denoted by ABHS, with respect to $K$ if

$$
\liminf _{\substack{\|x\| \rightarrow+\infty \\ x \in K}}\langle x, f(x)\rangle=+\infty
$$

The relations between conditions (BHS) and (ABHS) is given by the following result.

Proposition 4.1. Let $K \subset \mathbf{R}^{n}$ be a closed pointed convex cone and $f: \mathbf{R}^{n} \rightarrow \mathbf{R}^{n}$ a continuous function. If $f$ satisfies condition (ABHS), then $f$ satisfies condition (BHS). If $f$ satisfies condition (BHS), then the function $F(x)=f\left(T_{\rho}(x)+\left\|x-T_{\rho}(x)\right\| x\right.$ satisfies condition (ABHS).

Proof. We suppose that $f$ satisfies condition (ABHS), i.e., we have that

$$
\liminf _{\substack{\|x\| \rightarrow+\infty \\ x \in K}}\langle x, f(x)\rangle=+\infty
$$

In this case, given $r>0$, there exists $\rho>0$ such that for any $x \in K$ with $\|x\|=\rho$ we have $\langle x, f(x)\rangle>r$. Indeed, if this is not true, then for any $n \in \mathbf{N}$, there exists $x_{n} \in K$ with $\left\|x_{n}\right\|=n$ such that $\left\langle x_{n}, f\left(x_{n}\right)\right\rangle \leq r$. Therefore, condition (ABHS) is not satisfied. This contradiction implies that $f$ satisfies condition (BHS). Conversely, if $f$ satisfies condition (BHS), then by Remark 3.3 F satisfies condition (ABHS).

Corollary 4.1. If $K \subset \mathbf{R}^{n}$ is a closed pointed convex cone and $f: \mathbf{R}^{n} \rightarrow \mathbf{R}^{n}$ is a continuous function satisfying condition (ABHS), then $N C P(f, K)$ has a solution. 
Proof. This corollary is a consequence of Proposition 4.1 and Theorem 3.3.

In the following we will show that condition (ABHS) is a good mathematical tool for the study of properties (a) and (b) of the interior band mapping $\mathcal{U}$ with respect to the cone $\mathbf{R}_{+}^{n}$.

In regards to the existence of a solution to the $N C P(f, K)$ in $r^{n}$ we cite also the following result.

Proposition 4.2. Let $K \subset \mathbf{R}^{n}$ be a closed pointed convex cone and $f: \mathbf{R}^{n} \rightarrow \mathbf{R}^{n}$ a continuous function. If $f$ satisfies the condition

$$
\liminf _{\substack{\|x\| \rightarrow \infty \\ x \in K}}\langle x, f(x)\rangle>0
$$

then $N C P(f, K)$ has a solution.

Proof. It is sufficient to prove that $f$ is without EFE with respect to $K$. Indeed, if we suppose that $f$ has an EFE, namely, $\left\{x^{r}\right\}_{r>0} \subset K$, then we have

$$
\left\langle x^{r}, f\left(x^{r}\right)\right\rangle=\left\langle x^{r}, u^{r}-\mu_{r} x^{r}\right\rangle=\left\langle x^{r}, u^{r}\right\rangle-\mu_{r}\left\langle x^{r}, x^{r}\right\rangle=-\mu_{r}\left\|x^{r}\right\|^{2}<0,
$$

which implies

$$
\liminf _{\left\|x^{r}\right\| \rightarrow \infty}\left\langle x^{r}, f\left(x^{r}\right)\right\rangle \leq 0
$$

This relation is impossible because we supposed condition (5).

Theorem 4.1. If $f: \mathbf{R}_{+}^{n} \rightarrow \mathbf{R}^{n}$ is a continuous function, then

$$
\liminf _{\substack{\|x\| \rightarrow \infty \\ x \in \mathbf{R}_{++}^{+}}}\langle x, f(x)\rangle=+\infty
$$

if and only if

$$
\liminf _{\substack{\|x\| \rightarrow \infty \\ x \in \mathbf{R}_{+}^{n}}}\langle x, f(x)\rangle=+\infty
$$


Proof. Obviously if

$$
\liminf _{\substack{\|x\| \rightarrow \infty \\ x \in \mathbf{R}_{+}^{n}}}\langle x, f(x)\rangle=+\infty
$$

then

$$
\liminf _{\substack{\|x\| \rightarrow \infty \\ x \in \mathbf{R}_{++}^{n}}}\langle x, f(x)\rangle=+\infty .
$$

The converse follows if we show that if

$$
\liminf _{\substack{\|x\| \rightarrow \infty \\ x \in \mathbf{R}_{++}^{n}}}\langle x, f(x)\rangle=+\infty
$$

then

$$
\liminf _{\substack{\|x\| \rightarrow \infty \\ x \in \partial \mathbf{R}_{+}^{n}}}\langle x, f(x)\rangle=+\infty
$$

Let $\left\{x^{n}\right\}$ be a sequence such that $\left\|x^{n}\right\| \rightarrow \infty$ as $n \rightarrow \infty$ and for any $n \in \mathbf{N}, x^{n} \in \partial \mathbf{R}_{+}^{n}$. Let $n$ be fixed. Since $x^{n} \in \partial \mathbf{R}_{+}^{n}$, there exists $\left\{y^{m}\right\} \subset \mathbf{R}_{++}^{n}$ such that $\left\{y^{m}\right\} \rightarrow x^{n}$ as $m \rightarrow \infty$. For any $n \in \mathbf{N}$ we select such a sequence $\left\{y^{m}\right\}$. Let $\varepsilon_{0}>0$ be an arbitrary real number. For any $n \in \mathbf{N}$ we can select the sequence $\left\{y^{m}\right\}$ such that $\left\|y^{m}-x^{n}\right\|<\varepsilon_{0}$, for any $m \in \mathbf{N}$. We can suppose that for any $n \in \mathbf{N}$, $\left\|f\left(x^{n}\right)\right\|>0$. Because $\lim _{m \rightarrow \infty}\left\|x^{n}-y^{m}\right\|=0$, there exists $m_{1} \in \mathbf{N}$ such that

$$
\left\|x^{n}-y^{m}\right\|<\frac{1}{\left\|f\left(x^{n}\right)\right\|},
$$

for any $m>m_{1}$.

Because $f$ is continuous, there exists an $m_{2} \in \mathbf{N}$ such that

$$
\left\|f\left(x^{n}\right)-f\left(y^{m}\right)\right\|<\frac{1}{\varepsilon_{0}+\left\|x^{n}\right\|}
$$

for any $m>m_{2}$. For any $m>\max \left\{m_{1}, m_{2}\right\}$ we have

$$
\left\|y^{m}\right\| \leq\left\|x^{n}-y^{m}\right\|+\left\|x^{n}\right\|<\varepsilon_{0}+\left\|x^{n}\right\|
$$


and

$$
\left\|f\left(x^{n}\right)-f\left(y^{m}\right)\right\|<\frac{1}{\varepsilon_{0}+\left\|x^{n}\right\|}<\frac{1}{\left\|y^{m}\right\|} .
$$

We take an arbitrary $m>\max \left\{m_{1}, m_{2}\right\}$ and we have

$$
\left\|x^{n}-y^{m}\right\|<\frac{1}{\left\|f\left(x^{n}\right)\right\|}
$$

and

$$
\left\|f\left(x^{n}\right)-f\left(y^{m}\right)\right\|<\frac{1}{\left\|y^{m}\right\|} .
$$

If we denote by $y^{n, *}=y^{m}$, then we have

$$
\left\|x^{n}-y^{n, *}\right\|<\frac{1}{\left\|f\left(x^{n}\right)\right\|}
$$

and

$$
\left\|f\left(x^{n}\right)-f\left(y^{n, *}\right)\right\|<\frac{1}{\left\|y^{n, *}\right\|} .
$$

We have

$$
\left\langle y^{n, *}, f\left(y^{n, *}\right)\right\rangle=\left[\left\langle y^{n, *}, f\left(y^{n, *}\right)\right\rangle-\left\langle x^{n}, f\left(x^{n}\right)\right\rangle\right]+\left\langle x^{n}, f\left(x^{n}\right)\right\rangle
$$

and

$$
\begin{aligned}
\|\left\langle y^{n, *}, f\left(y^{n, *}\right)\right\rangle & -\left\langle x^{n}, f\left(x^{n}\right)\right\rangle \| \\
& \leq\left\|\left\langle x^{n}-y^{n, *}, f\left(x^{n}\right)\right\rangle\right\|+\left\|\left\langle y^{n, *}, f\left(x^{n}\right)-f\left(y^{n, *}\right)\right\rangle\right\| \\
& <\frac{1}{\left\|f\left(x^{n}\right)\right\|} \cdot\left\|f\left(x^{n}\right)\right\|+\left\|y^{n, *}\right\| \cdot \frac{1}{\left\|y^{n, *}\right\|}=2 .
\end{aligned}
$$

Therefore,

$$
\left\langle y^{n, *}, f\left(y^{n, *}\right)\right\rangle<2+\left\langle x^{n}, f\left(x^{n}\right)\right\rangle
$$

Since $\left\|x^{n}\right\|-\left\|y^{n, *}\right\| \leq\left\|x^{n}-y^{n, *}\right\|<\varepsilon_{0}$, for any $n \in \mathbf{N}$, we have $\left\|x^{n}\right\|<\varepsilon_{0}+\left\|y^{n, *}\right\|$, for any $n \in \mathbf{N}$, which implies $\left\|y^{n, *}\right\| \rightarrow+\infty$, because $\left\|x^{n}\right\| \rightarrow+\infty$. 
Computing liminf in (6), we have

$$
\liminf _{n \rightarrow \infty}\left\langle x^{n}, f\left(x^{n}\right)\right\rangle=+\infty
$$

Therefore,

$$
\liminf _{\substack{\|x\| \rightarrow+\infty \\ x \in \partial \mathbf{R}_{+}^{n}}}\langle x, f(x)\rangle=+\infty
$$

and the proof is complete.

5. Scalar derivatives. Let $C \subseteq \mathbf{R}^{n}$ be a set which contains at least one nonisolated point: $F: \mathbf{R}^{n} \rightarrow \mathbf{R}^{n}$ and $x_{0}$ a nonisolated point of $C$. The following definition is an extension of Definition $2.2[\mathbf{2 6}]$ :

Definition 5.1. The limit

$$
\underline{F}^{\#, C}\left(x_{0}\right)=\liminf _{\substack{x \rightarrow x_{0} \\ x \in C}} \frac{\left\langle F(x)-F\left(x_{0}\right), x-x_{0}\right\rangle}{\left\|x-x_{0}\right\|^{2}}
$$

is called the lower scalar derivative of $f$ at $x_{0}$ along $C$. Taking limsup in place of liminf, we can define the upper scalar derivative $\bar{F}^{\#, C}\left(x_{0}\right)$ of $F$ at $x_{0}$ along $C$ similarly.

Scalar derivatives were studied in $[\mathbf{2 6}, \mathbf{2 7}]$ and successfully applied to fixed point theorems in $[\mathbf{1 9}, \mathbf{2 0}]$.

6. Inversions. The following definition can be found in $[\mathbf{7}$, Example 5.1, page 169]:

Definition 6.1. The operator

$$
i: \mathbf{R}^{n} \backslash\{0\} \longrightarrow \mathbf{R}^{n} \backslash\{0\} ; \quad i(x)=\frac{x}{\|x\|^{2}}
$$

is called inversion (of pole 0 ).

It is easy to see that $i$ is one to one and $i^{-1}=i$. 
Let $f: \mathbf{R}^{n} \rightarrow \mathbf{R}^{n}$ be a mapping. Since $\mathbf{R}^{n} \backslash\{0\}$ is an invariant set of $i$, the following definition makes sense.

Definition 6.2. The inversion (of pole 0) of the mapping $f$ is the mapping $\mathcal{I}(f): \mathbf{R}^{n} \rightarrow \mathbf{R}^{n}$ defined by:

$$
\mathcal{I}(f)(x)= \begin{cases}\|x\|^{2}(f \circ i)(x) & \text { if } x \neq 0 \\ 0 & \text { if } x=0\end{cases}
$$

It is easy to see that the inversion of mappings $\mathcal{I}$ is a one to one operator on the set of mappings $\left\{f \mid f: \mathbf{R}^{n} \rightarrow \mathbf{R}^{n} ; f(0)=0\right\}$ and $\mathcal{I}^{-1}=\mathcal{I}$, i.e., $\mathcal{I}(\mathcal{I}(f))=f$.

The properties of inversions were studied in detail in [19].

\section{Infinitesimal interior point $\varepsilon$ exceptional families.}

Definition 7.1. Let $g: \mathbf{R}^{n} \rightarrow \mathbf{R}^{n}$ be a function. Given a scalar $\varepsilon>0$, we say that a sequence $\left\{y^{r}\right\}_{r>0} \subset \mathbf{R}_{++}^{n}$ is an infinitesimal interior point $\varepsilon$ exceptional family for $g$ if $\left\|y^{r}\right\| \rightarrow 0$ as $r \rightarrow+\infty$ and for each $y^{r}$ there exists a positive number $0<\mu_{r}<1$ such that

$$
g_{l}\left(y^{r}\right)=\frac{1}{2}\left(\mu_{r}-\frac{1}{\mu_{r}}\right) y_{l}^{r}+\frac{\varepsilon \mu_{r}}{y_{l}^{r}}\left\|y^{r}\right\|^{4},
$$

for all $l=1,2, \ldots, n$.

Proposition 7.1. If $f: \mathbf{R}^{n} \rightarrow \mathbf{R}^{n}$ is a continuous function and $g=\mathcal{I}(f)$ is the inversion of $f$, then $\left\{x^{r}\right\} \subset \mathbf{R}_{++}^{n}$ is an interior point $\varepsilon$-exceptional family for $f$ if and only if $\left\{y^{r}\right\} \subset \mathbf{R}_{++}^{n}$ is an infinitesimal interior point $\varepsilon$-exceptional family for $g$, where $y^{r}=i\left(x^{r}\right)$ is the inversion of $x^{r}$, for all $r>0$.

Proof. Suppose that $\left\{x^{r}\right\}_{r>0} \subset \mathbf{R}_{++}^{n}$ is an interior point $\varepsilon$-exceptional family for $f$, and let

$$
y^{r}=i\left(x^{r}\right),
$$


for all $r>0$. Since $i^{-1}=i$, equations (2) and (8) imply that

$$
f_{l}\left(i\left(y^{r}\right)\right)=\frac{1}{2}\left(\mu_{r}-\frac{1}{\mu_{r}}\right) i\left(y^{r}\right)_{l}+\frac{\varepsilon \mu_{r}}{i\left(y^{r}\right)_{l}},
$$

for all $l=1,2, \ldots, n$. Multiplying both sides of equation (9) by $\left\|y^{r}\right\|^{2}$ we obtain equation (7). Hence, $\left\{y^{r}\right\}_{r>0} \subset \mathbf{R}_{++}^{n}$ is an infinitesimal interior point $\varepsilon$-exceptional family for $g$. Similarly it can be proved that if $\left\{y^{r}\right\}_{r>0} \subset \mathbf{R}_{++}^{n}$ is an infinitesimal interior point $\varepsilon$-exceptional family for $g$, then $\left\{x^{r}\right\}_{r>0} \subset \mathbf{R}_{++}^{n}$ is an interior point $\varepsilon$-exceptional family for $f$.

Theorem 7.1. Let $f: \mathbf{R}^{n} \rightarrow \mathbf{R}^{n}$ be a continuous function and $\varepsilon>0$. If there is no infinitesimal interior point $\varepsilon$-exceptional family for $g=\mathcal{I}(f)$, then there exists a point $x(\varepsilon)$ such that

$$
x(\varepsilon)>0, f(x(\varepsilon))>0, x_{l}(\varepsilon) f_{l}(x(\varepsilon))=\varepsilon,
$$

for all $l=1,2, \ldots, n$.

Proof. Suppose, to the contrary, that there is no point $x(\varepsilon)$ which satisfies relation (10). Then, by Theorem 2.1, the function $f$ has an interior point $\varepsilon$-exceptional family $\left\{x^{r}\right\}_{r>0} \subset \mathbf{R}_{++}^{n}$. Hence, Proposition 7.1 implies that $\left\{y^{r}\right\}_{r>0} \subset \mathbf{R}_{++}^{n}$ is an infinitesimal interior point $\varepsilon$-exceptional family for $g$, where $y^{r}=i\left(x^{r}\right)$, for all $r>0$. But this is in contradiction with our assumption.

By Theorem 7.1 it is interesting to find conditions under which the inversion of a continuous function does not possess an infinitesimal interior point $\varepsilon$-exceptional family for all $\varepsilon>0$. For such functions $\mathcal{U}(\varepsilon) \neq \varnothing$, for each $\varepsilon>0$.

\section{Results related to properties (a) and (b) of the interior band mapping $\mathcal{U}$.}

Theorem 8.1. Let $f: \mathbf{R}^{n} \rightarrow \mathbf{R}^{n}$ be a continuous function. If

$$
\liminf _{\substack{\|x\| \rightarrow+\infty \\ x \in \mathbf{R}_{++}^{n}}}\langle x, f(x)\rangle=+\infty
$$


then

(1) the problem $N C P\left(f, \mathbf{R}_{+}^{n}\right)$ has a solution,

(2) $\mathcal{U}(\varepsilon) \neq \varnothing$, for any $\varepsilon>0$,

(3) for any fixed $\varepsilon_{0}>0$ the set $\cup_{\left.\varepsilon \in] 0, \varepsilon_{0}\right]} \mathcal{U}(\varepsilon)$ is bounded.

Proof. (1) By Theorem 4.1 we have that

$$
\liminf _{\substack{\|x\| \rightarrow+\infty \\ x \in \mathbf{R}_{+}^{n}}}\langle x, f(x)\rangle=+\infty
$$

and by Proposition 4.1 we have that $f$ satisfies condition (BHS). Applying Theorem 3.3 we obtain that $N C P\left(f, \mathbf{R}_{+}^{n}\right)$ has a solution.

(2) By using Theorem 2.1 it is sufficient to show that $f$ does not have an interior point $\varepsilon$-exceptional family $\left\{x^{r}\right\}_{r>0} \subset \mathbf{R}_{++}^{n}$. Indeed, we suppose that $f$ has an interior point $\varepsilon$-exceptional family $\left\{x^{r}\right\}_{r>0} \subset$ $\mathbf{R}_{++}^{n}$. Multiplying formula (2) given in Definition 2.1 by $x_{l}^{r}$ and summing with $l$ from 1 to $n$, we obtain

$$
\left\langle x^{r}, f\left(x^{r}\right)\right\rangle=\frac{1}{2}\left(\mu_{r}-\frac{1}{\mu_{r}}\right)\left\|x^{r}\right\|^{2}+n \varepsilon \mu_{r},
$$

where $0<\mu_{r}<1$, for any $r>0$. From the last equality we deduce

$$
\left\langle x^{r}, f\left(x^{r}\right)\right\rangle+\frac{1}{2}\left(\frac{1}{\mu_{r}}-\mu_{r}\right)\left\|x^{r}\right\|^{2}<n \varepsilon .
$$

Let $r_{0}>0$ such that $\left\|x^{r_{0}}\right\|>0$. Because $\left\|x^{r}\right\| \rightarrow+\infty$ as $r \rightarrow+\infty$, we can consider a subsequence $\left\{x^{r_{i}}\right\}$ such that $\left\|x^{r_{0}}\right\|<\left\|x^{r_{i}}\right\|$ and $\left\|x^{r_{i}}\right\| \rightarrow+\infty$ as $i \rightarrow \infty$. For this subsequence we have

$$
\frac{1}{2}\left(\frac{1}{\mu_{r_{i}}}-\mu_{r_{i}}\right)\left\|x^{r_{0}}\right\|^{2}+\left\langle x^{r_{i}}, f\left(x^{r_{i}}\right)\right\rangle<n \varepsilon .
$$

Computing liminf and using the assumption of our Theorem, we obtain a contradiction. Therefore, by Theorem 2.1, we have that $\mathcal{U}(\varepsilon) \neq \varnothing$, for any $\varepsilon>0$.

(3) We observe that for any $x(\varepsilon) \in \mathcal{U}(\varepsilon)$ we have $\langle x(\varepsilon), f(x(\varepsilon))\rangle=n \varepsilon$. Now, we suppose that there is an $\varepsilon>0$ such that $\cup_{\left.\varepsilon \in] 0, \varepsilon_{0}\right]} \mathcal{U}(\varepsilon)$ is not 
bounded. Then, there is a sequence $x\left(\varepsilon_{k}\right) \in \mathcal{U}\left(\varepsilon_{k}\right)$ with $\left.\left.\varepsilon_{k} \in\right] 0, \varepsilon_{0}\right]$ which is not bounded. Hence, by the assumption of our theorem we have

$$
\liminf _{k \rightarrow \infty}\left\langle x\left(\varepsilon_{k}\right), f\left(x\left(\varepsilon_{k}\right)\right)\right\rangle=+\infty .
$$

On the other hand,

$$
\left\langle x\left(\varepsilon_{k}\right), f\left(x\left(\varepsilon_{k}\right)\right)\right\rangle=n \varepsilon_{k} \leq n \varepsilon_{0},
$$

which implies

$$
\liminf _{k \rightarrow \infty}\left\langle x\left(\varepsilon_{k}\right), f\left(x\left(\varepsilon_{k}\right)\right)\right\rangle \leq n \varepsilon_{0}
$$

and we have a contradiction. Therefore, $\cup_{\left.\varepsilon \in] 0, \varepsilon_{0}\right]} \mathcal{U}(\varepsilon)$ is bounded for all $\varepsilon_{0}>0$.

We remark that in Theorem 8.1 conclusion (1) is (a) of $\mathcal{U}$ and conclusion (2) is property (b).

Theorem 8.2. Let $f: \mathbf{R}^{n} \rightarrow \mathbf{R}^{n}$ be a continuous function If

$$
\liminf _{\substack{\|x\| \rightarrow+\infty \\ x \in \mathbf{R}_{++}^{n}}} \frac{\langle x, f(x)\rangle}{\|x\|^{2}}>0,
$$

then the interior band mapping $\mathcal{U}$ has properties (a) and (b).

Proof. We denote by

$$
r=\liminf _{\substack{\|x\| \rightarrow+\infty \\ x \in \mathbf{R}_{++}^{n}}} \frac{\langle x, f(x)\rangle}{\|x\|^{2}}
$$

and we take $r_{0}$ such that $0<r_{0}<r$. There exists $\rho>0$ such that for any $x \in \mathbf{R}_{++}^{n}$ with $\|x\|>\rho$,

$$
\frac{\langle x, f(x)\rangle}{\|x\|^{2}}>r_{0} .
$$


Indeed if this is not true, then for any $n \in \mathbf{N}$, there exists $x^{n} \in \mathbf{R}_{++}^{n}$ with $\left\|x^{n}\right\|>n$ and such that

$$
\frac{\left\langle x^{n}, f\left(x^{n}\right)\right\rangle}{\left\|x^{n}\right\|^{2}} \leq r_{0} .
$$

Because $\left\|x^{n}\right\| \rightarrow+\infty$ as $n \rightarrow \infty$ we have that

$$
\liminf _{n \rightarrow \infty} \frac{\left\langle x^{n}, f\left(x^{n}\right)\right\rangle}{\left\|x^{n}\right\|^{2}} \leq r_{0}<r,
$$

which is impossible. Therefore, for any $x \in \mathbf{R}_{++}^{n}$ with $\|x\|>\rho$ we have $\langle x, f(x)\rangle>r_{0}\|x\|^{2}$ which implies that

$$
\liminf _{\substack{\|x\| \rightarrow+\infty \\ x \in \mathbf{R}_{++}^{n}}}\langle x, f(x)\rangle=+\infty .
$$

Applying Theorem 8.1 we obtain that the interior band mapping $\mathcal{U}$ has properties (a) and (b).

Theorem 8.3. Let $f: \mathbf{R}^{n} \rightarrow \mathbf{R}^{n}$ be a continuous function and $g=\mathcal{I}(f)$. If the lower scalar derivative of $g$ in 0 along $\mathbf{R}_{++}^{n}$ is positive, then the interior band mapping $\mathcal{U}$ has properties (a) and (b).

Proof. We have

$$
\underline{g}^{\#}(0)=\liminf _{\substack{y \rightarrow 0 \\ y \in \mathbf{R}_{++}^{n}}} \frac{\langle g(y), y\rangle}{\|y\|^{2}}
$$

Let $y=i(x)$. Then, we have

$$
\liminf _{\substack{y \rightarrow 0 \\ y \in \mathbf{R}_{++}^{n}}} \frac{\langle g(y), y\rangle}{\|y\|^{2}}=\liminf _{\substack{\|x\| \rightarrow \infty \\ x \in \mathbf{R}_{++}^{n}}} \frac{\langle f(x), x\rangle}{\|x\|^{2}} .
$$

Equations (11) and (12) imply

$$
\underline{g}^{\#}(0)=\liminf _{\substack{\|x\| \rightarrow \infty \\ x \in \mathbf{R}_{++}^{n}}} \frac{\langle f(x), x\rangle}{\|x\|^{2}} .
$$

Hence, the result follows by using Theorem 8.1. 
The following definition extends the notion of copositive functions, see [17]. We remark that the notion can be introduced along an arbitrary convex cone in a Hilbert space too, but for our investigation it is sufficient to consider the case of $\mathbf{R}^{n}$ with the cone $\mathbf{R}_{++}^{n}$.

Definition 8.1. The function $f: \mathbf{R}^{n} \rightarrow \mathbf{R}^{n}$ is called asymptotically copositive along $\mathbf{R}_{++}^{n}$ if there is a $\rho>0$ such that

$$
\langle f(x), x\rangle \geq 0
$$

for all $x \in \mathbf{R}_{++}^{n}$ with $\|x\|>\rho$.

The following definition can be formulated along an arbitrary convex cone in a Hilbert space too, but for the same reason as above we would consider the case of $\mathbf{R}^{n}$ with the cone $\mathbf{R}_{++}^{n}$ only.

Definition 8.2. The function $f: \mathbf{R}^{n} \rightarrow \mathbf{R}^{n}$ is called strongly asymptotically copositive along $\mathbf{R}_{++}^{n}$ if there are $\beta, \rho>0$ such that

$$
\langle f(x), x\rangle \geq \beta\|x\|^{2},
$$

for all $x \in \mathbf{R}_{++}^{n}$ with $\|x\|>\rho$.

We remark that $f$ is strongly asymptotically copositive along $\mathbf{R}_{++}^{n}$ if and only if there is a $\beta>0$ such that the function $f-\beta I$ is asymptotically copositive along $\mathbf{R}_{++}^{n}$, where $I$ is the identity function of $\mathbf{R}^{n}$.

The following theorem follows directly from Theorem 8.1 and Definition 8.2.

Theorem 8.4. If $f: \mathbf{R}^{n} \rightarrow \mathbf{R}^{n}$ is a continuous strongly asymptotically copositive function along $\mathbf{R}_{++}^{n}$, then the interior band mapping $\mathcal{U}$ has properties (a) and (b).

Corollary 8.1. Let $f: \mathbf{R}^{n} \rightarrow \mathbf{R}^{n}$ be a continuous function. If there is $a \rho>0$ and $\beta>0$ such that $f(x)-\beta x \in \mathbf{R}_{+}^{n}$, for all $x \in \mathbf{R}_{++}^{n}$ with $\|x\|>\rho$, then the interior band mapping $\mathcal{U}$ has properties (a) and (b). 
Proof. We have

$$
\langle f(x)-\beta x, x\rangle \geq 0
$$

for all $x \in \mathbf{R}_{++}^{n}$ with $\|x\|>\rho$. Hence,

$$
\langle f(x), x\rangle \geq \beta\|x\|^{2},
$$

for all $x \in \mathbf{R}_{++}^{n}$ with $\|x\|>\rho$. Therefore, $f$ is strongly asymptotically copositive along $\mathbf{R}_{++}^{n}$ and the result follows from Theorem 8.4.

At the end of our paper we present two results: Theorem 8.5 and Corollary 8.3, which show that the coercivity condition of Theorem 8.2 can be satisfied by a large class of functions. For this we need the following corollary which is a particularization of Corollary 3.1 [19]:

Corollary 8.2. Let $D=\left\{x \in \mathbf{R}^{n}:\|x\| \leq 1\right\}$ and $f: \mathbf{R}^{n} \rightarrow \mathbf{R}^{n}$; $f(0)=0$. There exists a unique extension $\tilde{f}: \mathbf{R}^{n} \rightarrow \mathbf{R}^{n}$ of $\left.f\right|_{D}$ such that $\tilde{f}$ is a fixed point of $\mathcal{I}$, i.e., $\tilde{f}=\mathcal{I}(\tilde{f})$.

We proved in our paper $[\mathbf{1 9}]$ that this extension has the form

$$
\tilde{f}(x)= \begin{cases}f(x) & \text { if }\|x\| \leq 1 \\ \mathcal{I}(f)(x) & \text { if }\|x\|>1\end{cases}
$$

Since $\tilde{f}=\mathcal{I}(\tilde{f})$ and

$$
\liminf _{\|x\| \rightarrow \infty} \frac{\langle\tilde{f}(x), x\rangle}{\|x\|^{2}}=\underline{\mathcal{I}(\tilde{f})}^{\#}(0) \quad(\text { by }[\mathbf{1 9}, \text { Lemma 4.1]), }
$$

we have

$$
\liminf _{\|x\| \rightarrow \infty} \frac{\langle\tilde{f}(x), x\rangle}{\|x\|^{2}}=\underline{\tilde{f}}^{\#}(0)=\underline{f}^{\#}(0)
$$

Hence,

$$
\liminf _{\|x\| \rightarrow \infty} \frac{\langle\tilde{f}(x), x\rangle}{\|x\|^{2}}>0
$$

if and only if

$$
\underline{f}^{\#}(0)>0 \text {. }
$$


On the other hand, by using the Cauchy inequality it is easy to see that if $\tilde{f}$ satisfies condition (13), then for any $b \in \mathbf{R}^{n}, \tilde{f}+b$ also satisfies condition (13). Hence, we have the following result:

Theorem 8.5. Let $b \in \mathbf{R}^{n}, f: \mathbf{R}^{n} \rightarrow \mathbf{R}^{n}$ with $f(0)=0$ and $\underline{f}^{\#}(0)>0, \tilde{f}: \mathbf{R}^{n} \rightarrow \mathbf{R}^{n}$ with

$$
\tilde{f}(x)= \begin{cases}f(x) & \text { if }\|x\| \leq 1 \\ \mathcal{I}(f)(x) & \text { if }\|x\|>1\end{cases}
$$

and $F=\tilde{f}+b$. Then, $F$ satisfies the condition

$$
\liminf _{\|x\| \rightarrow \infty} \frac{\langle F(x), x\rangle}{\|x\|^{2}}>0 .
$$

Corollary 8.3. Let $a, b \in \mathbf{R}^{n}, f: \mathbf{R}^{n} \rightarrow \mathbf{R}^{n}$ with $f(0)=a$ and $\underline{f}^{\#}(0)>0$, and $F: \mathbf{R}^{n} \rightarrow \mathbf{R}^{n}$ with

$$
F(x)= \begin{cases}f(x)-a+b & \text { if }\|x\| \leq 1 \\ \mathcal{I}(f)(x)-\|x\|^{2} a+b & \text { if }\|x\|>1\end{cases}
$$

Then, $F$ satisfies the condition

$$
\liminf _{\|x\| \rightarrow \infty} \frac{\langle F(x), x\rangle}{\|x\|^{2}}>0 .
$$

Proof. Let $f_{0}(x)=f(x)-a$. It is easy to see that $f_{0}(0)=0$ and $\underline{f}_{0}^{\#}(0)=\underline{f}^{\#}(0)>0$. Hence, we can apply Theorem 8.5 to the function $\overline{f_{0}}$ to obtain the desired result.

For $A: \mathbf{R}^{n} \rightarrow \mathbf{R}^{n}$ a linear operator, we denote by $A_{s}$ the operator $\left(A+A^{*}\right) / 2$, where $A^{*}$ is the adjoint of $A$. Let $\sigma\left(A_{s}\right)$ be the spectrum of $A_{s}$. With these notations we have as follows:

Remark 8.1. If $f$ satisfies supplementary conditions in 0 , the paper [27] provides useful computational formulae for checking the condition $\underline{f}^{\#}(0)>0$ : 
(1) By Theorem $1.1[\mathbf{2 7}]$ if $f$ is locally Lipschitz in 0 and the directional derivative $f^{\prime}(0 ; h)$ exists for each $h$, then

$$
\underline{f}^{\#}(0)=\inf _{\|h\|=1}\left\langle f^{\prime}(0 ; h), h\right\rangle
$$

(2) By Theorem $1.2[\mathbf{2 7}]$ if $f$ is Frechét differentiable in 0, with the differential $d f(0)$, then

$$
\underline{f}^{\#}(0)=\min _{\|h\|=1}\langle d f(0)(h), h\rangle
$$

(3) By Theorem $1.5[\mathbf{2 7}]$ if $f$ is Frechét differentiable in 0 with the differential $d f(0)$, then

$$
\underline{f}^{\#}(0)=\min \sigma((d f(0)))_{s} .
$$

9. Comments. In this paper we studied the interior band of $\varepsilon$-solutions of the nonlinear complementarity problem defined by a continuous function from $\mathbf{R}^{n}$ to $\mathbf{R}^{n}$ and by the cone $\mathbf{R}_{+}^{n}$.

By the results presented in this paper we put in evidence the importance of the asymptotic Browder Hartman Stampacchia condition. By using this condition and the scalar derivative, we obtained some new results related to the interior band of $\varepsilon$-solutions.

By our method we do not need to suppose that the mapping $f$ is uniformly $P$-mapping or monotone mapping as in several papers cited in our references.

Our ideas presented in this paper may be a starting point for new developments.

\section{REFERENCES}

1. J. Burke and $\mathrm{S} . \mathrm{Xu}$, The global linear convergence of a non-interior-point path following algorithm for linear complementarity problems, Math. Oper. Res. 23 (1998), 719-734.

2. - A non-interior predictor-corrector path following algorithm for the monotone linear complementarity problem, Math. Program. 87 (2000), 113-130.

3. B. Chen and X. Chen, A global and local superlinear continuation-smoothing method for $P_{0}$ and $R_{0} N C P$ or monotone NCP, SIAM J. Optim. 9 (1999), 624-645. 
4. B. Chen, X. Chen and C. Kanzow, A generalized Fischer-Burmeister NCPfunction: Theoretical investigation and numerical results, Technical Report, Zur Angewandten Mathematik, Hamburger Beiträge, 1997.

5. C. Chen and O.L. Mangasarian, A class of smoothing functions for nonlinear and mixed complementarity problems, Comput. Optim. Appl. 5 (1996), 97-138.

6. R.W. Cottle, J.S. Pang and R.E. Stone, The linear complementarity problem, Academic Press, Boston 1992.

7. M.P. do Carmo, Riemannian geometry, Birkhäuser, Boston, 1992.

8. F. Facchini, Structural and stability properties of $P_{0}$ nonlinear complementarity problems, Math. Oper. Res. 23 (1998), 735-749.

9. F. Facchini and C. Kanzow, Beyond monotonicity in regularization methods for nonlinear complementarity problems, SIAM J. Control Optim. 37 (1999), $1150-1161$.

10. M.C. Ferris and J.S. Pang, Engineering and economic applications of complementarity problems, SIAM Rev. 39 (1997), 669-713.

11. M.S. Gowda and M.A. Tawhid, Existing and limiting behavior of trajectories associated with $P_{0}$-equations, Comput. Optim. Appl. 12 (1999), 229-251.

12. O. Güler, Existence of interior points and interior-point paths in nonlinear monotone complementarity problems, Math. Oper. Res. 18 (1993), 128-147.

13. - Path following and potential reduction algorithm for nonlinear monotone complementarity problems, Technical Report, Department of Management Sciences, The University of Iowa, Iowa City, 1990.

14. P.T. Harker and J.S. Pang, Finite-dimensional variational inequality and nonlinear complementarity problems: A survey of theory, Algorithms Appl., Math. Program. 48 (1990), 161-220.

15. K. Hotta and A. Yoshise, Global convergence of a class of non-interior-point algorithms using Chen-Harker-Kanzow functions for nonlinear complementarity problems, Math. Program. 86 (1999), 105-133.

16. G. Isac, Complementarity problems, Lecture Notes in Math. 1528, SpringerVerlag, Berlin, 1992.

17. Topological methods in complementarity theory, Kluwer Academic Publishers, 2000.

18. G. Isac, V. Bulavski and V. Kalashnikov, Exceptional families, topological degree and complementarity problems, J. Global Optim. 10 (1997), 207-225.

19. G. Isac and S.Z. Németh, Scalar derivatives and scalar asymptotic derivatives. Properties and some applications, J. Math. Anal. Appl. 278 (2003), 149-170.

20. - Scalar derivatives and asymptotic scalar derivatives. An Altman type fixed point theorem on convex cones and some applications, J. Math. Anal. Appl., in print.

21. C. Kanzow, Some nonlinear continuation methods methods for nonlinear complementarity problems, SIAM J. Matrix Anal. Appl. 17 (1996), 851-868.

22. M. Kojima, N. Megiddo and T. Noma, Homotopy continuation methods for nonlinear complementarity problems, Math. Oper. Res. 16 (1991), 754-774. 
23. M. Kojima, N. Megiddo, T. Noma and A. Yoshise, A unified approach to interior point algorithms for linear complementarity problems, Lecture Notes Comput. Sci. 538, Springer-Verlag, 1991.

24. N. Megiddo, Pathways to the optimal set in nonlinear programming, in Progress in mathematical programming: Interior-point and related methods, $\mathrm{N}$. Megiddo, ed., Springer-Verlag, New York, 1989.

25. R.D.C. Monteiro and I. Adler, Interior path following primal dual algorithms, Part I: Linear programming, Math. Program. 44 (1989), 27-42.

26. S.Z. Németh, A scalar derivative for vector functions, Rivista di Matematica Pura ed Applicata 10 (1992), 7-24.

27. - Scalar derivatives and spectral theory, Mathematica, 35 (1993), 49-58.

28. P. Tseng, An infeasible path-following method for monotone complementarity problems, SIAM J. Optim. 7 (1997), 386-402.

29. Y.B. Zhao and G. Isac, Properties of a multivalued mapping associated with some nonmonotone complementarity problems, SIAM J. Control Optim. 39 (2000), $571-593$.

Department of Mathematics and Computer Science, Royal Military College of Canada, P.O. Box 17000, STN Forces Kingston, Ontario K7K 7B4, Canada

Email address: isac-g@rmc.ca

The University of Birmingham, School of Mathematics, Watson BuildING, B15 2TT BiRMingham, United Kingdom

Email address: nemeths@for.mat.bham.ac.uk 